\title{
Supporting Information for Implications of alternative substrate binding modes for catalysis by uracil-DNA glycosylase: An apparent discrepancy resolved
}

\author{
Ao Ma, Jie Hu, Martin Karplus and Aaron R. Dinner
}

August 18, 2006

\begin{tabular}{llrllr}
\hline index & type & scaling factor & index & residue & scaling factor \\
\hline 2 & Met & 36.50 & 6 & Glu & 65.36 \\
9 & Lys & 41.49 & 10 & Lys & 70.42 \\
15 & Glu & 12.87 & 18 & Lys & 23.58 \\
23 & Lys & 43.67 & 30 & Glu & 16.64 \\
33 & Lys & 28.25 & 52 & Asp & 26.46 \\
54 & Lys & 29.24 & 55 & Asp & 38.17 \\
57 & Lys & 18.45 & 81 & Arg & 11.51 \\
94 & Lys & 4.50 & 95 & Glu & 7.20 \\
99 & Asp & 9.62 & 101 & Glu & 70.92 \\
102 & Asp & 50.51 & 116 & Lys & 22.22 \\
146 & Asp & 7.33 & 170 & Lys & 4.76 \\
171 & Lys & 4.13 & 176 & Asp & 42.74 \\
177 & Arg & 18.83 & 178 & Lys & 77.52 \\
179 & Arg & 29.67 & 201 & Arg & 25.25 \\
205 & Lys & 27.03 & 208 & Glu & 36.23 \\
212 & Lys & 51.55 & 215 & Lys & 22.78 \\
216 & Lys & 65.36 & 219 & Asp & 19.84 \\
221 & Lys & 30.77 & 222 & Glu & 23.58 \\
225 & Thy & 1.17 & 226 & Ura & 1.33 \\
227 & Ade & 1.36 & 228 & Thy & 2.46 \\
\hline
\end{tabular}

Table S.1: Scaling factors for ionizable residues and phosphate groups in the ssDNA simulations. Each scale factor is the ratio of the potential at the origin from a specific residue in vacuum $(\epsilon=1)$ and that in aqueous solution ( $\epsilon=1$ for the protein and $\epsilon=80$ for the solution). The origin was taken to be the grid site closest to the $N_{\epsilon}$ atom of $\mathrm{H} 148$. 


\begin{tabular}{llrllr}
\hline index & type & scaling factor & index & residue & scaling factor \\
\hline 2 & Met & 36.50 & 6 & Glu & 65.36 \\
9 & Lys & 41.49 & 10 & Lys & 70.92 \\
15 & Glu & 12.87 & 18 & Lys & 23.58 \\
23 & Lys & 43.67 & 30 & Glu & 16.72 \\
33 & Lys & 28.25 & 52 & Asp & 26.46 \\
54 & Lys & 29.24 & 55 & Asp & 38.31 \\
57 & Lys & 18.48 & 81 & Arg & 11.55 \\
94 & Lys & 4.55 & 95 & Glu & 7.21 \\
99 & Asp & 9.62 & 101 & Glu & 70.92 \\
102 & Asp & 50.25 & 116 & Lys & 22.27 \\
146 & Asp & 7.33 & 170 & Lys & 4.69 \\
171 & Lys & 4.13 & 176 & Asp & 42.74 \\
177 & Arg & 18.76 & 178 & Lys & 77.52 \\
179 & Arg & 29.67 & 201 & Arg & 25.25 \\
205 & Lys & 27.03 & 208 & Glu & 36.23 \\
212 & Lys & 51.55 & 215 & Lys & 22.83 \\
216 & Lys & 65.36 & 219 & Asp & 19.88 \\
221 & Lys & 30.86 & 222 & Glu & 23.64 \\
225 & Gua & 1.63 & 226 & Thy & 1.18 \\
227 & Ura & 1.34 & 228 & Ade & 1.37 \\
229 & Thy & 2.44 & 230 & Cyt & 6.22 \\
231 & Thy & 20.33 & 232 & Thy & 36.36 \\
234 & Ade & 4.16 & 235 & Ade & 3.56 \\
236 & Gua & 5.29 & 237 & Ade & 10.47 \\
238 & Thy & 15.24 & 239 & Ade & 10.74 \\
240 & Ade & 7.28 & 241 & Cyt & 8.14 \\
242 & Ade & 6.55 & & & \\
\hline & & & & &
\end{tabular}

Table S.2: Scale factors for ionizable residues and phsophate groups in the dsDNA simulations. 